\title{
Rapid mass segregation in young star clusters without substructure?
}

\author{
C. Olczak ${ }^{1,2,3}$, R. Spurzem ${ }^{4,2,3}$, Th. Henning ${ }^{1}$ \\ ${ }^{1}$ Max-Planck-Institut für Astronomie, Heidelberg, Germany \\ email: olczak@mpia.de \\ ${ }^{2}$ National Astronomical Observatories of China, Chinese Academy of Sciences, Beijing, China \\ ${ }^{3}$ The Kavli Institute for Astronomy and Astrophysics at Peking University, Beijing, China \\ ${ }^{4}$ Astronomisches Rechen-Institut, Universität Heidelberg, Heidelberg, Germany
}

\begin{abstract}
The young star clusters we observe today are the building blocks of a new generation of stars and planets in our Galaxy and beyond. Despite their fundamental role we still lack knowledge about the initial conditions under which star clusters form and the impact of these often harsh environments on the formation and evolution of their stellar and substellar members.

We present recent results showing that mass segregation in realistic models of young star clusters occurs very quickly for subvirial spherical systems without substructure. This finding is a critical step to resolve the controversial debate on mass segregation in young star clusters and provides strong constraints on their initial conditions. The rapid concentration of massive stars is usually associated with strong gravitational interactions early on during cluster evolution and the subsequent formation of multiple systems and ejection of stars.
\end{abstract}

Keywords. stellar dynamics, methods: n-body simulations, galaxies: star clusters

\section{Introduction}

It is commonly accepted that star formation does usually not occur in isolation but that a large majority of young stars - up to $90 \%$ - are part of a cluster (e.g. Lada \& Lada 2003). One of the most widely discussed aspect of the dynamical evolution of young star clusters is that of mass segregation (e.g. Raboud \& Mermilliod 1998; Bonnell \& Davies 1998; Gouliermis et al. 2004; Chen et al. 2007; Vesperini et al. 2009; Xin-Yue et al. 2009; Ascenso et al. 2009). A major question is whether the observed degree of mass segregation has developed dynamically (via two-body encounters) or whether it can be only explained via primordial mass segregation (i.e. inherent to the star formation process).

Though it has been shown that mass segregation in a stellar system with a mass spectrum (e.g. Spurzem \& Takahashi 1995; Khalisi et al. 2007) occurs rapid enough to account for the observed mass segregation, recent investigations have focused on how substructure in stellar systems can accelerate this process (McMillan et al. 2007; Allison et al. 2009; Moeckel \& Bonnell 2009, see also (Aarseth \& Hills 1972)). However, all these works have simulated the evolution under subvirial initial conditions.

We thus investigate numerically the pure effect of subvirial conditions in young star clusters and infer to which degree they can speed-up mass segregation.

\section{Numerical Setup}

We have performed numerical simulations of isolated, non-rotating, moderately populated star clusters with 1000 stars initially, a Kroupa IMF in the range $0.08 \mathrm{M}_{\odot}$ to $50 \mathrm{M}_{\odot}$ Kroupa (2001), a density profile $\rho \propto r^{-2}$ and varying initial virial ratios $Q$. We 

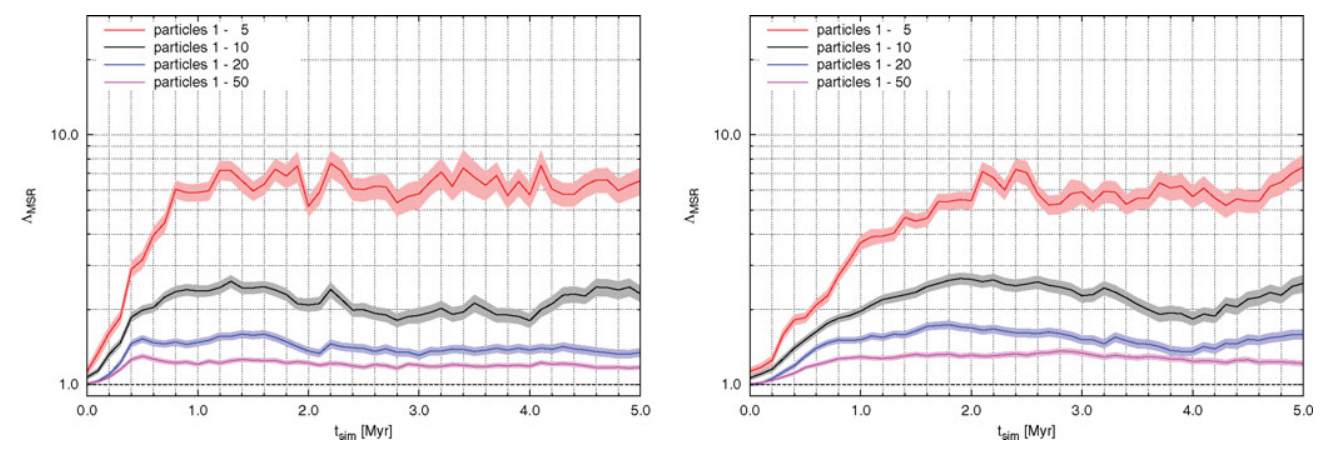

Figure 1. Evolution of mass segregation in a star cluster with $Q=0.1$ (left) and $Q=0.3$ (right) represented by $\Lambda_{\mathrm{MST}}$ of four different stellar groups (from top to bottom: 5, 10, 20, and 50 most massive stars). The shaded regions indicate the associated uncertainties. One dynamical crossing time corresponds to $0.19 \mathrm{Myr}$ for $Q=0.1$ and $0.28 \mathrm{Myr}$ for $Q=0.3$.

use the NBody6-GPU code Aarseth (2003) to model the dynamics of these numerical models and analyze the degree of mass segregation, $\Lambda_{\mathrm{MST}}$, via a minimum spanning tree algorithm as described by Allison et al. (2009).

\section{Results}

We find that mass segregation occurs rapidly in star clusters with subvirial initial conditions (see Fig. 1). If $Q=0.1$, the maximum degree of segregation is reached in less than 1 Myr (or roughly five crossing times). The five most massive stars show strong mass segregation with $\Lambda_{\mathrm{MST}} \approx 7$. The first evidence of significant mass segregation, $\Lambda_{\mathrm{MST}} \gtrsim 2$, occurs even within 1-2 crossing times. For less cold initial conditions $(Q=0.3)$ the evolution is about two times slower yet still very rapid and equally pronounced.

The concentration of massive stars is accompanied by strong gravitational interactions that involve early on the formation of multiple systems and ejection of (massive) stars. This counteracts further mass segregation and quickly limits its maximum degree.

Compared with the time scale on which mass segregation occurs in the simulations of McMillan et al. (2007) and Allison et al. (2009) the present setup leads to an equally rapid evolution. This indicates that in fact subvirial initial conditions might be the dominant dynamical driver of rapid mass segregation in young star clusters.

\section{References}

Aarseth, S., 2003, Gravitational N-body Simulations (Cambridge, Cambridge University Press, 2003,430 p.). Aarseth, S. J., \& J. G. Hills, 1972, A\&A 21, 255.

Allison, R. J., S. P. Goodwin, R. J. Parker, R. de Grijs, S. F. Portegies Zwart, \& M. B. N. Kouwenhoven, 2009, ApJ 700, L99.

Ascenso, J., J. Alves, \& M. T. V. T. Lago, 2009, A\&A 495, 147.

Bonnell, I. A., \& M. B. Davies, 1998, MNRAS 295, 691.

Chen, L., R. de Grijs, \& J. L. Zhao, 2007, AJ 134, 1368.

Gouliermis, D., S. C. Keller, M. Kontizas, E. Kontizas, \& I. Bellas-Velidis, 2004, A\&A 416, 137.

Khalisi, E., P. Amaro-Seoane, \& R. Spurzem, 2007, MNRAS 374, 703.

Kroupa, P., 2001, MNRAS 322, 231.

Lada, C. J. \& E. A. Lada, 2003, ARA\&A 41, 57

McMillan, S. L. W., E. Vesperini, \& S. F. Portegies Zwart, 2007, ApJ 655, L45.

Moeckel, N. \& I. A. Bonnell, 2009, MNRAS, 13700908.0253.

Raboud, D. \& J.-C. Mermilliod, 1998, A\&A 333, 897.

Spurzem, R. \& K. Takahashi, 1995, MNRAS 272, 772.

Vesperini, E., S. L. W. McMillan, \& S. Portegies Zwart, 2009, ApJ 698, 615.

Xin-Yue, E., J. Zhi-Bo \& F. Yan-Ning, 2009, Chinese Astronomy and Astrophysics 33, 139. 